\title{
An ethnographic approach to oral assessment in French as a foreign language
}

\section{[Une approche ethnographique de l'évaluation de l'oral en français langue étrangère]}

\author{
Yiru Xu - Hsin-I Lee
}

DOI: 10.18355/XL.2018.11.01XL.22

\begin{abstract}
The present article proposes a methodological discussion about oral evaluation in the domain of French as a foreign language. We adopt an ethnographic perspective which aims at observing, understanding, interpreting and analyzing the phenomena which take place during a sequence in order to identify the interactional and contextual characteristics of the situation. This situation requires, a series of preparations regarding choice and access to the terrain, on-site observation, recording method, researcher attitude and data processing. This methodological approach also allows us to nourish a reflection on this foreign language pedagogical context.
\end{abstract}

Key words: oral evaluation, ethnographic approach, terrain, foreign language teaching

\section{Résumé}

Le présent article propose une discussion méthodologique dans la situation de l'évaluation de l'oral en français langue étrangère. Nous adoptons une perspective ethnographique qui vise à observer, comprendre, interpréter et analyser les phénomènes qui ont lieu au cours d'une séquence afin de relever les caractéristiques interactionnelles et contextuelles de la situation. Pour ce faire, une suite de préparations en amont est exigée par la situation en ce qui concerne le choix et l'accès au terrain, l'observation sur place, la méthode d'enregistrement, la posture du chercheur et le traitement des données. Cette démarche méthodologique nous permet également d'enrichir une réflexion sur ce contexte didactique en langues étrangères.

Mots-clés : évaluation de l'oral, approche ethnographique, terrain, FLE

\section{Introduction}

Le terme évaluer, en ancien français (IV $\left.{ }^{\mathrm{e}}\right)$ avaluer (1283), met l'accent sur value, qui signifie «fixer la valeur de »1. En moyen français, ce terme conserve le sens de "fixer le prix, la valeur de », mais aussi "déterminer approximativement par une appréciation la valeur de quelque chose $»^{2}$. Actuellement, selon Le petit Robert, ce terme regroupe deux sens: l'un signifie «porter un jugement sur la valeur, l'importance » et l'autre «fixer approximativement». Ce survol étymologique nous montre un paradoxe: on évalue pour porter un jugement sur la valeur de quelque chose, mais on ne peut que fixer approximativement cette valeur puisque la subjectivité existe toujours.

Cette préoccupation demeure dans tout type d'évaluation et notamment dans celle de l'oral. Ceci peut se traduire par une complexité de la relation entre l'interaction et le

1. Dictionnaire de l'ancienne langue française et de tous ses dialectes du IXème au XVème siècle, Frédéric Godefroy, 1880-1895. http://micmap.org/dicfro/introduction/dictionnaire-godefroy

2. Dictionnaire du Moyen Français (1330-1500). http://www.atilf.fr/dmf

XLinguae, Volume 11 Issue 1XL, January 2018, ISSN 1337-8384, eISSN 2453-711X 
contexte. D'une part, l'évaluation se concentre sur la qualité et le résultat de l'interaction; celle-ci constitue l'objet que l'évaluateur observe en vue de prendre une décision. D'autre part, les processus d'évaluation se réalisent par le biais de l'interaction ; celle-ci constitue donc également le médium de l'épreuve en évaluation en face à face. Par conséquent, parmi toutes les situations d'évaluation, celle de l'oral représente une caractéristique plus complexe et épineuse, car elle constitue un processus dans lequel l'évaluateur doit réaliser simultanément plusieurs tâches : la collecte des données, l'analyse des informations, la prise de décision, tout en participant parfois à l'interaction. En outre, étant donné le caractère éphémère de l'oral combiné au fait que l'évaluation peut se présenter comme une sorte de « sanction ", l'interaction est conditionnée par des facteurs environnementaux, psychologiques et relationnels de la situation que Coste and Moore (1992) appellent un « lieu de malaise ».

C'est pourquoi, adopter une approche qui permet de rendre cette situation intelligible et de mettre en lumière certaines caractéristiques de cette situation de face à face, nous parait naturel et primordial dans la recherche sur l'évaluation de l'oral. Nous voudrions, dans cet article, aborder une discussion méthodologique sous un angle à la fois théorique et empirique dans une perspective ethnographique en didactique des langues étrangères. Nous commencerons par un choix épistémologique de cette approche dans le domaine de l'éducation et plus spécifiquement dans celui de la didactique. Ensuite, nous traiterons ce sujet en détail dans le contexte d'évaluation en mettant l'accent sur les difficultés du terrain avant d'aborder le contexte de notre étude. Enfin, nous expliquerons, étape par étape, la démarche de la constitution des données et nos réflexions.

\section{La perspective ethnographique en didactique des langues}

Dans le domaine de la didactique, les premières recherches qui s'intéressent à la méthodologie de l'analyse des interactions verbales apparaissent dans les pays anglosaxons où les chercheurs envisagent la « classroom research » selon trois orientations (Allwright et Bailey, 1991) : la première est la «theory first »; les chercheurs font des hypothèses issues d'une réflexion théorique. La collecte des données et les analyses sont organisées dans le but de vérifier ces hypothèses ou de répondre à la question de départ. La deuxième orientation, "data first ", va dans le sens contraire, elle se base sur l'analyse des données afin de faire surgir les questions et de construire la théorie. La troisième, principalement développée dans les recherches en sciences de l'éducation et en didactique, est l'" action research », qui est issue des problèmes rencontrés dans la classe par les enseignants ou par les apprenants. Les chercheurs veulent les étudier avec l'objectif de trouver des solutions ou améliorations. Ainsi, les recherches débutent souvent par une identification de la problématique qui est suivie par une recherche de connaissances et d'informations. Puis, en fonction de cette problématique, les chercheurs vont aller sur le terrain ou mettre en place des dispositifs didactiques et pédagogiques afin de les tester.

En didactique du français langue étrangère, les recherches se situent, depuis une trentaine années, dans le prolongement de ces orientations anglo-saxonnes. Elles cherchent «des formes multiples de réalisation des innovations 》 (Canelas Trevisi et al., 1999 : 144) qui se fondent sur une observation détaillée, de nature qualitative, dans les contextes naturels, et les modifient le moins possible. Ceci s'inscrit dans une perspective ethnographique qui «a une préférence manifeste pour les données empiriques recueillies dans les contextes naturels, et se donne une multiplicité de méthodes et de perspectives pour atteindre ses objectifs » (Cambra Giné, 2003 : 97). Les finalités de la démarche ethnographique en éducation visent à comprendre et interpréter les phénomènes qui ont lieu dans le milieu de la classe. Cambra Giné résume l'intérêt de cette approche empirique dans une classe de langue sous un triple aspect : «d'une part, analyser le discours éducatif pour comprendre les processus 
d'interaction pédagogique au service de la construction progressive de significations et de savoirs partagés ; d'autre part, examiner ce discours produit par la communication et la construction sociale de la connaissance dans ce qu'il révèle sur l'appropriation des langues par les apprenants ; finalement, découvrir les manifestations des volontés sous-jacentes des participants, contrainte par leur système de représentations » (Cambra Giné, 2003 : 15).

Cette démarche est à la fois empirique et «naturaliste » dans le sens où les sources de données ne sont pas fabriquées ni manipulées, mais sont issues du contexte réel où se produisent les événements. Ainsi, le contexte s'avère essentiel dans la compréhension de l'interaction entre les participants, qui permet de situer les évènements dans leur cadre spatio-temporel et socio-culturel. Par conséquent, la focalisation sur le contexte de l'interaction didactique se révèle une option cruciale et légitime dans les recherches interprétatives (Van Lier, 1988).

\section{L'évaluation : un terrain de haute tension}

Avec cette perspective ethnographique, le choix du contexte didactique devient notre première préoccupation dans la collecte des données. Cette démarche implique pour le chercheur de se déplacer sur le terrain pour observer ce qui se passe. Cependant, il n'est jamais facile de convaincre une institution, un enseignant d'ouvrir la porte à qui peut lui paraître un intrus (Cicurel, 2011a). Dans le cadre de notre projet de recherche, nous nous sommes heurtées de nombreuses difficultés.

Lorsque nous avons choisi de mener notre recherche sur les situations d'évaluation, la constitution d'un corpus semblait nécessaire voire obligatoire dans cette démarche pour nous permettre de les observer et de les analyser. Néanmoins, l'évaluation, qui diffère d'autres situations didactiques comme celles de la classe par exemple, représente une situation délicate et épineuse. En effet, les fonctions d'évaluation sont à la fois diagnostique, formative et sommative, c'est-à-dire que l'évaluation constitue non seulement un bilan de l'apprentissage qui sert à classer et à sélectionner les apprenants, mais devient aussi une finalité qui oriente l'enseignement (Meirieu, 1990) et assure l'articulation entre les caractéristiques des apprenants et celles du système de formation (Allal, 1979). Par conséquent, les acteurs de l'enseignement et de l'éducation (enseignants, apprenants, encadrants, parents) sont en demande d'une évaluation exacte, transparente et accordant à la note une valeur symbolique et fiable (Huver \& Spinger, 2011). L'évaluation subit la pression conjointe de l'institution, de la société et même du monde économique dans lesquels les participants se trouvent. Leur pratique est en outre marquée par l'insécurité dans un environnement global compétitif qui prescrit l'excellence et l'échec.

Cette pression s'accroit en évaluation de l'oral qui se présente comme un contexte sensible, source de tension pour les participants et complexe au niveau interactionnel et communicatif. En situation d'évaluation de l'oral, les dimensions émotionnelles, psychologiques, affectives et corporelles sont présentes, pour le candidat comme pour l'évaluateur. Les situations d'évaluation le révèlent davantage que celles de classe. Tout cela détermine la difficulté d'accès à ce type de terrain au niveau à la fois institutionnel, didactique et méthodologique. Cette inaccessibilité du terrain devient notre plus grande difficulté dans la récolte de données. Mais notre visée étant d'observer la situation authentique de l'évaluation, les simulations en l'absence d'enjeux institutionnels et interactionnels ne correspondent évidemment pas à notre objectif. En effet, seules les données qui ne sont ni arrangées ni orchestrées par les chercheurs peuvent fidèlement représenter les comportements réels des participants et les particularités du contexte (Mondada, 2009). Nous n'avons donc pas adopté la méthode de reconstitution.

XLinguae, Volume 11 Issue 1XL, January 2018, ISSN 1337-8384, eISSN 2453-711X 


\section{Terrain de la recherche}

Nous avons finalement fixé notre terrain de recherche au Centre International d'Études Françaises (CIEF) pour plusieurs raisons : il est spécialisé dans la formation linguistique et culturelle d'un public non francophone et membre de l'Association des Directeurs de Centres Universitaires d'Études Françaises pour Étrangers (A.D.C.U.E.F.E.)/Campus FLE qui attribue les Diplômes Universitaires d'Études Françaises (DUEF). Ces diplômes sont reconnus dans différents centres membres en France et permettent aux apprenants de poursuivre leur formation en langue française et éventuellement de s'inscrire dans un cursus universitaire. Ainsi, le résultat d'évaluation apporte une valeur institutionnelle et même sociale aux apprenants qui prennent en considération l'importance de l'évaluation. De plus, le centre propose tous types de formations semestrielles et annuelles de tous les niveaux (A1, A2, B1, $\mathrm{B} 2, \mathrm{C} 1$ et $\mathrm{C} 2$ selon le CECR) sur toutes les compétences (compréhension orale et écrite, expression orale et écrite ainsi que la formation spécialisée), ce qui nous offre une possibilité de sélectionner un niveau et une compétence précis afin de mener notre recherche. Par ailleurs, les formations sont ouvertes aux étudiants étrangers non francophones de toutes les nationalités, quel que soit leur niveau en français, sous condition qu'ils soient majeurs et titulaires d'un diplôme équivalent au baccalauréat. Ceci nous permet d'avoir un public relativement homogène en ce qui concerne son profil et ses besoins.

\subsection{Public}

Les apprenants qui ont effectué leur inscription administrative en formation sont étudiants de l'université et doivent participer à tous les examens proposés et réussir afin d'obtenir leur diplôme. Pendant la formation, plusieurs contrôles continus et finaux sont effectués pour chaque cours. À la fin du semestre, le diplôme de niveau est calculé en fonction des résultats obtenus dans ces contrôles. Avec ce type de fonctionnement institutionnel, le terrain nous guide pour choisir une classe à suivre sur toutes sortes de situations de contrôle. Cette classe est composée de 13 étudiants dont 11 chinois et 2 coréens, leur âge se situe entre 19 et 28 ans. Ils suivent une formation en FLE pendant 12 semaines, soit 280 heures, dans les compétences suivantes : compréhension orale et écrite, expression orale et écrite, phonétique et civilisation.

La plupart d'entre eux ont déjà une base de français avant d'arriver en France. Après avoir passé un test d'entrée, ils débutent leur cursus par le niveau A2. Les raisons du choix de ce niveau sont doubles : en premier lieu, il s'agit d'un niveau qu'on pourrait qualifier de "faux débutant », avec lequel ils peuvent en principe s'engager dans la communication mais pour lequel il leur manque des connaissances langagières. De ce fait, pour réaliser des activités communicatives pendant l'évaluation, ils doivent recourir à diverses stratégies qu'ils exercent de manière plus explicite et plus visible que ne le font des apprenants du niveau intermédiaire ou avancé. La deuxième raison concerne le choix de la situation de communication. Pas de situation culturelle spécifique, en principe, toutes les situations sont habituelles et familières dans la majorité des sociétés et des contextes. Ainsi, les candidats peuvent toujours se servir, dans la communication en langue étrangère, des connaissances générales acquises dans leur langue maternelle. Deux cours d'expression orale sont proposés pour ce groupe. L'un se déroule en classe entière, il est focalisé sur des exercices en interaction (jeu de rôles) : savoir obtenir un objet ou un service, accepter ou refuser une invitation, accepter ou refuser une proposition, organiser une activité, échanger des informations... L'autre, en demi-classe, se concentre sur les thèmes suivants : parler de soi, d'un lieu, des gens, d'un objet, de ses goûts, etc.

\subsection{L'évaluateur}

Dans le cadre de l'évaluation orale, nous distinguons deux types d'évaluateurs : l'évaluateur qui n'a aucune connaissance du candidat (par exemple dans le cas du 
DELF, DALF ou TCF), ce qui en principe devrait être garant d'un résultat dénué de préjugé. Cependant, il existe des impondérables comme l'échantillonnage de tests ou de questions, qui en revanche empêche l'évaluateur d'avoir un jugement plus complet. À l'opposé, le deuxième type d'évaluateur connait l'étudiant avant l'évaluation. Cette situation se présente souvent à la fin de l'année scolaire où l'évaluateur, professeur du candidat, va évaluer l'acquisition du cours. De Landsheere (1974) nomme « examen interne "l'évaluation dans laquelle le maître note les élèves qui ont reçu son enseignement dans le cadre de la classe ; et « examen externe », l'évaluation dont les épreuves sont organisées et notées par des jurys indépendants des écoles.

Dans notre terrain de recherche, les deux enseignantes chargées des cours d'expression orale sont également évaluatrices des contrôles. Les deux évaluatrices ont passé un semestre avec cette classe ; aussi ont-elles une bonne connaissance des candidats. Cette connaissance entre les candidats et les évaluateurs est conditionnée par le contexte institutionnel ; d'un côté, il est difficile d'organiser des séances d'évaluation indépendamment des cours d'expression orale en raison du temps et de l'effectif, d'un autre côté, cela donne une possibilité de comparer les données recueillies pendant l'évaluation avec l'observation en classe afin de donner un résultat récurrent.

\section{La démarche du recueil des données}

C'est une étape critique dans notre recherche, car d'un côté, chaque séance d'évaluation est inscrite selon le calendrier pédagogique de l'institution ; ni l'enseignant ni le chercheur ne peut le modifier. Ceci demande la connaissance et la préparation en amont. D'un autre côté, l'évaluation de l'oral est éphémère ; une fois terminée, elle ne laisse aucune trace. Il exige donc un choix des moyens d'enregistrement de ces évaluations afin de pouvoir les analyser.

\subsection{Une préparation en amont}

Dans la démarche ethnographique, afin de démontrer les évènements authentiques in situ, l'enregistrement de l'interaction est considéré comme le principal moyen de marquer les détails en montrant comment les activités langagières sont organisées et gérées par les participants. Comme le souligne Sacks (1984), les données enregistrées permettent aux chercheurs de documenter l'interaction authentique et d'assurer une accessibilité permanente aux données. Les avantages de l'enregistrement sont, d'après Wittrock (1986, cité par Cambra Giné, 2003) et Van Lier (1988, cité par Cambra Giné, 2003), la possibilité d'effectuer une analyse complète et exhaustive, de délibérer, de réviser ses interprétations, et d'étudier également les évènements peu fréquents, qui sont tout aussi intéressants (Cambra Giné, 2003). C'est pourquoi mener une observation avec enregistrement nous parait adéquat dans notre recueil de données.

Or, à la différence des autres situations didactiques, l'évaluation s'avère un contexte sensible et confidentiel, les chercheurs ne peuvent, en aucun cas, entrer brutalement avec leur caméra pour enregistrer. "L'entrée sur le terrain et la présence sur le terrain en vue de préparer les enregistrements sont des moments importants du point de vue des relations de confiance nouées avec les partenaires de l'enquête. Les questions éthiques concernent la manière dont ces personnes sont traitées, le rapport entre ce qu'on leur dit de l'enquête, ce qu'elles en attendent, ce qu'elles en tirent ainsi que les effets positifs ou négatifs de l'enquête sur leur vie » (Mondada, 2004 : 14).

Ainsi, avant tout, demander une autorisation concernant le droit d'entrer sur le terrain pour l'observation, l'enregistrement et son exploitation à des fins scientifiques et pédagogiques apparaît comme essentiel dans la démarche. En effet, l'acceptation de l'enregistrement ou de l'observation est étroitement dépendante de la compréhension des finalités de recherche ; le chercheur doit donner le maximum d'informations aux

XLinguae, Volume 11 Issue 1XL, January 2018, ISSN 1337-8384, eISSN 2453-711X 
participants afin d'obtenir leur assentiment et leur autorisation. Pour ce faire, nous l'avons expliqué à l'institution et aux participants, en précisant l'objectif, le projet, le déroulement et l'exploitation scientifique de nos données afin d'obtenir leur accord.

La dernière étape dans cette phase de préparation consiste en le choix du matériel enregistré. "Les choix à opérer pour l'enregistrement sont guidés par des considérations de technique et de savoir-faire qui sont elles-mêmes dépendantes d'exigences théoriques et analytiques » (Mondada, $2004: 16$ ). Le choix du matériel est conditionné par notre terrain d'observation. Nous avons choisi une combinaison d'enregistreur audio et vidéo, soit un dictaphone et deux caméras. L'audio permet généralement d'obtenir une meilleure qualité de son que la vidéo, grâce à sa technologie et à sa proximité des participants, tant pour faciliter la transcription que pour mieux comprendre chaque participant. Les deux caméras nous permettent d'observer tous les participants de l'évaluation, le déroulement temporel, le cadre de participation et aussi des aspects non verbaux : proxémique, gestes, mouvements, attitudes, regards, actions, cadre physique, tout ce qui nous aide à rappeler et à mieux comprendre l'interaction verbale et à mener des analyses multimodales.

\subsection{Le filmage, le rôle du chercheur et la caméra}

Selon le calendrier institutionnel, quatre sessions d'évaluation de l'oral sont organisées pendant un semestre : contrôles continus et contrôles finaux des cours d'expression orale en classe entière et en demi-groupe. Le premier contrôle continu du cours en classe entière a lieu dans une salle de cours où les tables et les chaises sont fixées par terre ; cela complique le filmage tant au niveau du cadrage que de la prise de son. Nous avons choisi l'enregistrement audio et vidéo des candidats qui passent l'évaluation et aussi l'enregistrement vidéo de l'évaluatrice. L'épreuve se déroule sous forme de jeu de rôles entre candidats.

Le déroulement du deuxième contrôle continu s'avère plus compliqué. Il se compose de deux parties. La première consiste en un monologue suivi individuel entre le candidat et l'évaluatrice. Les interactions se déroulent en face à face, nous avons donc choisi d'utiliser deux caméras disposées en vis à vis de manière à capter l'activité de chacun des participants. Après le monologue suivi, la deuxième partie se compose de jeux de rôles en petits groupes devant toute la classe. Pour mieux enregistrer l'activité de la classe, une caméra installée au fond de la salle filme le jeu des acteurs et une autre, installée sur le devant, enregistre les réactions du public.

Quelques semaines plus tard ont lieu les contrôles finaux. Le premier concerne les jeux de rôle entre candidat et évaluatrice sans présence du public. L'évaluatrice et le candidat se mettent à l'angle d'une table, une caméra peut encadrer deux participants et l'intensité sonore est satisfaisante. Le deuxième se déroule sous forme de monologue suivi dans lequel les candidats entrent dans la salle l'un après l'autre pour effectuer une présentation individuelle avec l'évaluatrice. Nous pouvons présenter les données sous forme de tableau ci-dessous :

\begin{tabular}{|c|l|l|l|l|c||}
\hline Situation & Nature d'épreuve & \multicolumn{1}{|c|}{ Public } & \multicolumn{1}{|c|}{ Effectif } & Nature & Durée \\
\hline 1 & $\begin{array}{l}\text { Jeu de rôles entre } \\
\text { candidats }\end{array}$ & $\begin{array}{l}\text { Sans } \\
\text { public }\end{array}$ & $\begin{array}{l}13 \text { étudiants } \\
7 \text { groupes }\end{array}$ & $\begin{array}{l}\text { Contrôle } \\
\text { continu }\end{array}$ & $1 \mathrm{~h} \mathrm{30}$ \\
\hline 2 & $\begin{array}{l}\text { Jeu de rôles entre } \\
\text { candidats }\end{array}$ & $\begin{array}{l}\text { Devant } \\
\text { public }\end{array}$ & $\begin{array}{l}13 \text { étudiants } \\
6 \text { groupes }\end{array}$ & $\begin{array}{l}\text { Contrôle } \\
\text { continu }\end{array}$ & $1 \mathrm{~h}$ \\
\hline 3 & $\begin{array}{l}\text { Jeu de rôles entre } \\
\text { candidat } \\
\text { évaluatrice }\end{array}$ & $\begin{array}{l}\text { Sans } \\
\text { public }\end{array}$ & 13 étudiants & $\begin{array}{l}\text { Contrôle } \\
\text { final }\end{array}$ & $2 \mathrm{~h}$ \\
\hline 4 & Monologue suivi & $\begin{array}{l}\text { Sans } \\
\text { public }\end{array}$ & 13 étudiants & $\begin{array}{l}\text { Contrôle } \\
\text { continu }\end{array}$ & $1 \mathrm{~h}$ \\
\hline 5 & Monologuesuivi & $\begin{array}{l}\text { Sans } \\
\text { public }\end{array}$ & 13 étudiants & $\begin{array}{l}\text { Contrôle } \\
\text { final }\end{array}$ & $1 \mathrm{~h} 10$ \\
\hline
\end{tabular}


Les contrôles continus et les contrôles finaux valent chacun $50 \%$ du coefficient. Autrement dit, dans notre corpus les contrôles continus présentent plutôt une fonction sommative que formative, c'est la raison pour laquelle nous ne ferons pas de distinction dans les analyses qui viennent.

Pendant le filmage, la présence du chercheur est nécessaire pour assurer un bon déroulement de captation. Cependant, cette présence modifie involontairement la situation. Le paradoxe de l'observateur, d'après Labov (1976), est que tout observateur a pour mission d'observer des interactions dont il voudrait qu'elles se déroulent comme s'il n'était pas là. Néanmoins, avec la présence des caméras, cette minimisation s'avère presque impossible, nous observons souvent la prise en compte des caméras de la part de l'évaluatrice et des candidats. A titre d'exemple, dans la situation de jeu de rôles entre candidat et évaluatrice, à la fin de chaque séance d'épreuve, l'évaluatrice regarde systématiquement le dictaphone sur la table et interroge l'observatrice sur le fonctionnement des caméras. Du côté des candidats, la présence des enregistreurs se répercute également dans leur interaction. Voici une séquence d'ouverture d'un jeu de rôles entre candidats :

Yy: candidate $\mathrm{Jj}$ : candidate

Yy bon- bonjour mademoiselle

$\mathrm{Jj} \quad$ ah bonjour bonjour ah qu'est-ce que qu'est-ce que

Yy $\quad{ }^{\circ}$ da dian sheng ${ }^{\circ}$ (en chinois: parle plus fort)

Les candidates sont conscientes d'être enregistrées et font attention à hausser le volume de leur voix afin de faciliter l'enregistrement. Pour modifier le moins possible la situation enregistrée, Allwright et Bailey (1991) recommandent aux chercheurs de donner aux personnes filmées la possibilité de s'habituer à leur présence, c'est-à-dire d'établir un contact avec les participants avant et après le filmage. Dans ce type de situation didactique, l'observateur, d'après Cicurel (2011b), n'est pas un étranger sur le terrain de son observation, car il appartient à la communauté des enseignants, il partage une grande partie des préoccupations, des savoirs et des valeurs avec les acteurs observés. C'est un regard d'enseignant sur d'autres enseignants, il s'agit d'une interprétation faite par des pairs en s'appuyant sur les expériences qu'ils ont.

\subsection{La transcription des données}

Après le recueil de données, l'étape suivante consiste à les transcrire pour les transformer en corpus observable et intelligible. Néanmoins, la transcription ne se réduit pas à une simple question technique, elle est à la fois une activité sélective et une entreprise interprétative (Mondada, 2000). La transcription constitue en effet la première interprétation des données qui établit un passage entre les matériaux oraux et les textes écrits, au cours de laquelle le chercheur commence une première sélection des données « brutes » afin de constituer un corpus. Ce premier « tri » s'effectue en fonction des objectifs de recherche et permet de mieux cibler les phénomènes intéressants.

Dans notre transcription, nous notons l'épreuve, le candidat, le changement de locuteur, les chevauchements, la pause la tonalité, l'allongement de syllabe, l'hésitation, le rire, le geste, le regard, le mouvement et la mimique. Concrètement :

Nomination de découpage. Le découpage du corpus en évaluation se révèle relativement simple par rapport au corpus en classe, car il est déjà naturellement découpé par la passation de chaque candidat. Par conséquent, dans la transcription nous respectons cette manière de découper le corpus et le nommons ainsi : 


\begin{tabular}{||r|l||}
\hline \hline Codage & \multicolumn{1}{|c||}{ Situation } \\
\hline JDRSP_Groupe 1 & $\begin{array}{l}\text { Jeu de rôles entre candidats sans présence de public, le groupe } \\
\text { numéro 1 }\end{array}$ \\
\hline $\begin{array}{l}\text { JDRAP_Groupe } \\
1\end{array}$ & $\begin{array}{l}\text { Jeu de rôles entre candidats avec présence de public, le groupe } \\
\text { numéro1 }\end{array}$ \\
\hline $\begin{array}{l}\text { JDREC_Candidat } \\
1\end{array}$ & Jeu de rôles entre candidat et évaluatrice, le candidat numéro 1 \\
\hline MS 1_Candidat 1 & Monologue suivi 1, le candidat numéro 1 \\
\hline MS 2_Candidat 1 & Monologue suivi 2, le candidat numéro 1 \\
\hline
\end{tabular}

Nomination des participants. Le principe de la nomination des participants dans la transcription consiste en une anonymisation qui permet de rendre les personnes enregistrées non identifiables à la fois au début de chaque tour de parole et aussi pendant l'interaction. C'est pourquoi nous avons choisi des pseudos noms dans la transcription. Les deux évaluatrices sont nommées E1 et E2, et les candidats par deux lettres initiales comme $\mathrm{Xt}, \mathrm{Ch}, \mathrm{Hm}$.

Transcription phonétique. La transcription phonétique prend en compte le caractère oral dans le langage qui englobe les phénomènes verbaux, vocaux et prosodiques afin de démontrer les caractéristiques naturelles du langage et nous permettre de réaliser des analyses détaillées sur ces phénomènes. A titre d'exemple :

JDRAP_Groupe 1

$1 \mathrm{Ch}$ euh j'ai vu: j'ai vu une bizarre chose

$2 \mathrm{Xt}$ c'est vrai/ c'est c'est quoi/

$3 \mathrm{Ch} \quad$ [euh:

$4 \mathrm{Mx}$ [euh: euh:: euh: ce matin on euh: euh on a trava: euh on a travaillé dans notre magasin et euh et euh un extra: terrestre a euh et: et entré: à: dans notre magasin

Dans cet extrait nous pouvons observer l'hésitation de la candidate Mx par le biais des onomatopées « euh:: » avec l'allongement de syllabe, l'intonation montante de la candidate Xt pour poser des questions «c'est vrai/ », une pause qui dure 1.8 secondes ainsi qu'un chevauchement au début du T3 et T4. Transcrire ces phénomènes permet de dévoiler l'enchaînement du tour de parole, le discours de chaque participant et les aspects prosodiques en langue orale.

Aspect multimodal. Les aspects non verbaux sont également transcrits dans notre corpus. Nous combinons les descriptions des gestes, regards, mouvements et les rires avec les transcriptions verbales comme ci-dessous.

JDRAP_Groupe 2

Ar ah: d'accord ((rire de la classe)) bizarre aussi euh::

(3.0)((Ar s'approche et regarde Yy))

Yy d'a- d'a-

Ar c'est quoi ((pointe avec la main))

Yy d'abord je: je voudrais: faire euh d'abord je voudrais faire faire pipi

((rire de la classe))

L'avantage de l'enregistrement vidéo est de nous permettre d'observer tous les aspects de l'interaction. Si nécessaire, nous rajoutons également dans la transcription des captures d'écran afin de montrer plus clairement les phénomènes non verbaux. Avec ces critères, la transcription s'effectue en trois étapes. Nous avons commencé par une transcription " brute » sans nous soucier des aspects multimodaux. Ensuite, nous appliquons une convention de transcription. Puis nous confrontons à plusieurs 
reprises la nouvelle transcription avec l'enregistrement vidéo pour rajouter les éléments multimodaux. Une fois la transcription terminée, la dernière étape consiste à commenter la transcription en notant des commentaires en parallèle se rapportant principalement aux phénomènes langagiers et interactionnels, aux explications et aux descriptions du discours. Ces commentaires sont descriptifs et narratifs, de sorte que le lecteur a l'impression de voir une représentation de la scène de façon minutieuse et contextualisée (Cambra Giné, 2003). Ceci permet de mieux appréhender la transcription et de relever les éléments qui nous intéressent, ce qui constitue la première phase d'analyse du corpus.

\section{Conclusion}

L'approche ethnographique dans le domaine de didactique des langues vise à observer, comprendre, interpréter et analyser les phénomènes qui ont lieu au cours d'une séquence afin de relever les caractéristiques interactionnelles et contextuelles de la situation. L'évaluation de l'oral constitue un terrain sensible et épineux dans lequel les dimensions émotionnelles, psychologiques, communicatives et corporelles des participants sont présentes. C'est pourquoi, ce terrain de haute tension exige une préparation en amont pour l'accès à l'institution, la demande d'autorisation, l'observation sur place et le choix d'enregistrement. Cette étape prédétermine le résultat et la qualité des données recueillies par le chercheur. En aval, la transcription est la dernière étape qui consiste à transformer les données audio et/ou vidéo en corpus écrit afin de l'exploiter.

Cette démarche méthodologique déterminée par les caractéristiques du terrain nous permet de mettre l'accent sur plusieurs points : d'une part, cette situation qui se déroule traditionnellement derrière la porte close d'une salle d'évaluation, nous montre un chantier peu exploité par les chercheurs et les formateurs ; ceci constitue une des raisons pour lesquelles les recherches en évaluation de l'oral progressent peu dans la didactique du FLE. Cette stagnation des recherches en évaluation de l'oral nécessite donc une mise en lumière de la recherche sur ce terrain. D'autre part, étant donné sa complexité et sa sensibilité, un recueil de données bien préparé, réfléchi, maitrisé et rationalisé, en ce qui concerne à la fois la méthodologie et la pratique constituent un préalable nécessaire pour garantir une bonne démarche de ce type de terrain.

\section{Bibliographic references}

ALLAL, L. 1979. Stratégies d'évaluation formative : conception psycho-pédagogique et modalités d'application. In L. Allal, J. Cardinet, \& P. Perrenoud (Éds.), L'évaluation formative dans un enseignement différencié. Berne : Peter Lang, pp.153183.

ALLWRIGHT, D. - BAILEY, K. M. 1991. Focus on the Language Classroom. Cambridge : Cambridge University Press.

CAMBRA GINE, M. 2003. Une approche ethnographique de la classe de langue etrangcre. Paris : Didier.

CANELAS, T. et al. 1999. L'objet enseigné : vers une méthodologie plurielle d'analyse des pratiques d'enseignement en classe. Repères : recherches en didactique du français langue maternelle. (20), 143-163.

CICUREL, F. 2011a. Les interactions en situation d'enseignement-apprentissage: observer, transcrire, analyser. In P. BLANCHET \& P. CHARDENET (Éds). Guide pour la recherche en didactique des langues et des cultures. Paris: Éditions des archives contemporaines. pp. 337-344.

CICUREL, F. 2011b. Les interactions dans l'enseignement des langues : agir professoral et pratiques de classe. Paris : Didier. 
COSTE, D. - MOORE, D. (Éd.) 1992. Autour de l'évaluation de l'oral, Bulletin CILA. vol.55.

DE LANDSHEERE, G. 1974. Évaluation continue et examens : précis de docimologie. Paris : F. Nathan.

HUVER, E. - SPRINGER, C. 2011. L'évaluation en langues. Paris : Didier.

LABOV, W. 1976. Sociologie. Paris : Minuit.

MEIRIEU, P. 1990. Pédagogie et évaluation différenciées. In C. DELORME (Éd.), L'évaluation en question. CEPEC. Paris : ESF éditeur.

MONDADA, L. 2000. Les effets theoriques des pratiques de transcription. Linx. Revue des linguistes de l'universite Paris X Nanterre. (42), pp.131-146.

MONDADA, L. 2004. Indications utiles pour la rédaction scientifique. Recueil inédit, Université Lumière Lyon 2, Département Sciences du langage.

MONDADA, L. 2009. La production de l'intelligibilité de l'action: une approche multimodale des procédés de sélection des locuteurs dans les interactions en classe. Éla. Études de linguistique appliquée. (1), pp. 25-40.

SACKS, H. 1984. Notes on methodology. Structures of social action: Studies in conversation analysis, pp. 21-27.

VAN LIER, L. 1988. The classroom and the language learner: Ethnography and second- language classroom research. London: Longman.

Word : 5118

Characters : 34299 (19,05 standard pages)

Assoc. Prof. Dr. Yiru Xu

Faculty of Foreign languages

University Sun Yat-Sen

No. 135, Xingang Xi Road, Guangzhou,

510275, P. R. China

Laboratory ICAR UMR 5191

Ecole Normale Supérieure de Lyon - Site Descartes

15, Parvis René Descartes

69342 Lyon

France

delphinexu@hotmail.com

Assoc. Prof. Dr. Hsin-I Lee

Institute NanFang of University Sun Yat-Sen

510970 Guangzhou

China

Hsini.Lee79@gmail.com 